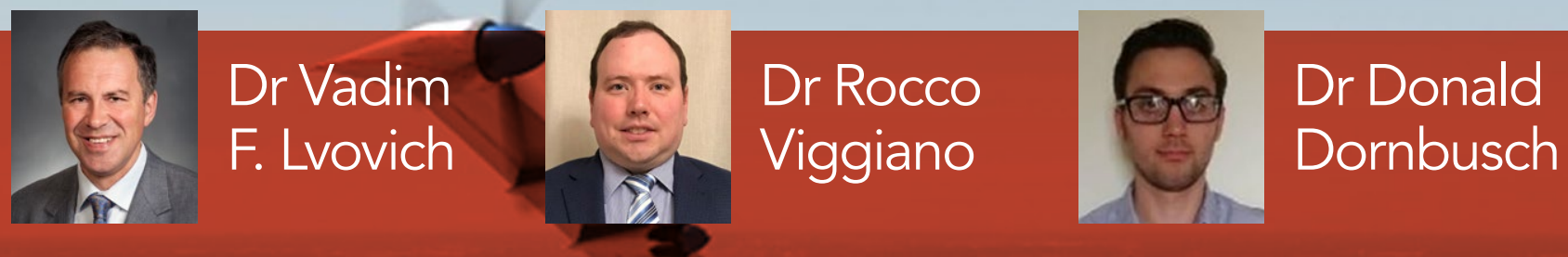

Office: +1 (216) 4335261 Cell: +1 (216) 9032151 E: Vadim.F.Lvovich@nasa.gov W: www.nasa.gov/aeroresearch/programs/tacp/cas

\title{
Monitoring electrolyte stability in lithium-air batteries for electric aircraft
}

\section{Detail}

Vadim F. Lvovich, PhD

Chief, Materials Chemistry and Physics Branch

NASA Glenn Research Center

21000 Brookpark Rd, 49-3 USA

Bio

Dr Vadim F. Lvovich has held several senior innovative technology management positions in industry, government and academia. He is currently Head of Materials Chemistry and Physics Branch at NASA Glenn Research Center. Dr Lvovich is an internationally renowned expert in theory and practice of electrochemical impedance spectroscopy.

Dr Rocco Viggiano has a PhD in Macromolecular Science and Engineering and currently leads NASA team in the development of solid-state battery technology to enable electric aviation.

Dr Donald Dornbusch has a PhD in Chemical Engineering. His experience encompasses materials for next generation energy storage focusing on beyond lithium-ion chemistries, including alternative cathodes, novel electrolytes, and high efficiency metal anodes.

\section{Funding}

Funding from Convergent Aeronautics Solution (CAS) Project of National Aeronautics and Space Administration (NASA) Aeronautics Research Mission Directorate (ARMD)

\section{Collaborators}

Dr John Lawson (NASA Ames Research Center), Dr Bryan McCloskey (University of California at Berkeley)

\section{Research Objectives}

Combining supercomputer modelling, fundamental chemistry analysis, advanced material science, and battery cell development to identify novel electrolyte components for advanced ultra-high energy batteries.

\section{References}

Dornbusch, D Viggiano, R Lvovich, V (2020). Integrated Impedance-NMR identification of electrolyte stability in Lithium-Air batteries. Electrochimica Acta. 349, 136169.

\section{Personal Response}

\section{Emissions from fossil fuels in aircraft are one of the main causes of global warming. When do you estimate that lithium-air battery technology could be safely made available at scale for electric aircraft?}

IV The lithium-oxygen technology still faces multiple challenges, including materials design, performance optimization of cells and components, cells scale-up, development of "balance of plant" for oxygen supply and by-products removal to and from the battery, and assembly of safe and efficient battery packs. Maturing of this technology requires addressing all these currently identified impediments, in addition to making lithium-oxygen technology commercially viable. If the history of current commercial lithium-ion battery technology is an indicator of speed of progress of a novel commercially viable scaled up battery technology, then the projection of introduction of lithium-oxygen technology may be optimistically put around years 2035-2040. 
Physical Sciences | Vadim Lvovich

\section{Monitoring electrolyte stability in lithium-air batteries for electric aircraft}

Switching to electric powered transport is one way of reducing $\mathrm{CO}_{2}$ emissions and their negative effects on the climate. Lithium-air batteries could play an important role in this transition, as they have the potential to provide the highest known theoretical energy density $(3,400 \mathrm{Wh} / \mathrm{kg})$. Development of lithium-air batteries requires highly stable electrolytes that are resistant to decomposition under operating conditions. Dr Donald Dornbusch, Dr Rocco Viggiano and Dr Vadim Lvovich from NASA are using a monitoring technique that uses Electrochemical
Impedance Spectroscopy (EIS) and Nuclear Magnetic Resonance (NMR) to unde Spectroscopy (ES) and Nulear Magnelic Resonance (NMR) highly stable battery components with an overarching goal of improving

E lectric aircraft are the way programme: an endeavour that aims to transform the global transportation system and meet the energy storage challenges of future aerospace missions.

NASA needs new battery materials to enable its vision, and lithium-air has the potential to provide batteries with the highest known theoretical energy density $(3,400 \mathrm{Wh} / \mathrm{kg})$. In physics, . system. In other words, a battery of a

given mass with a higher energy density will propel a vehicle

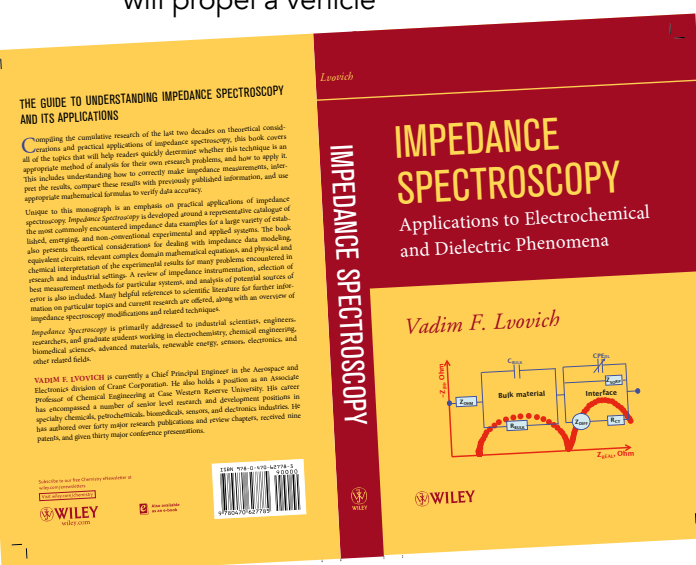

A balance of theoretical considerations and problem of identifying nove enallenging pactical problem solving of electrochemical components for advanced ultra- high energy batteries that can meet energy storage challenges of NASA The The researchers, based at the NASA John H. Glenn Research Center, USA, include Dr Vadim Lvovich, Dr Rocco Viggiano and Dr Donald Dornbusch. The team employs a technique Spectroscopy (EIS) together with Nuclear Magnetic Resonance (NMR), to monitor the Remical reations happening at the electrodes and to prevent and contrast the formation of decomposition products that could affect the efficiency and the number of charging cycles of lithium air batteries. EIS allows scientists to investigate the functioning of an electrochemical system, by applying an electric excitation signal and monitoring the resulting changes in flow of current. NMR is a very sensitive technique, which uses strong magnetic fields to identify the presence and structura Thegrity of molecules in a solution. The team uses NMR to identify the dolecules that are produced by

\section{REACTIONS AT THE ELECTRODES}

\section{IN LITHIUM AIR BATTERIES}

The team's recent paper (Dornbusch, generation in a lithium-oxygen cell as a multi-step process that involves three phases - gaseous (oxygen), liquid (electrolyte with lithium ions) and solid (carbon cathode). The oxygen, which is dissolved in the electrolyte, will react with lithium ions within the porous surface of the carbon electrode, yielding lithium peroxide $\left(\mathrm{L}_{2} \mathrm{O}_{2}\right)$. During the process, lithium carbonate $\left(\mathrm{L}_{2} \mathrm{CO}_{3}\right)$, formed as a side product, accumulates the surface of the electrode, reducing decomposition of the electrolyte.
The recent study also describes how the air cathode material and structure play Surface area and optimal porosity are important for both maintaining high power and achieving high energy. Electrodes with the same porosity but different pore size distribution show different performance due to the electrode passivation. For example, the low surface area of electrodes with larger pore sizes leads to a more severe electrode passivation. Similarly, the degradation of cathode morphology, caused by the decomposition of organic electrolytes, can lead to a severe decrease in the efficiency a of lithium-air batteries.

\section{DESIGN CRITERIA FOR NOVEL} In their roLTE CANDIDATES specify the criteria by which novel organic electrolytes for lithiumair batteries would be selected highlighting the importance of a medium that can dissolve high molar concentrations of lithium salt, oxyge and oxygen radicals. This is because lithium ions and solubilised oxygen are required for the reaction to occur at the cathode surface. Furthermore, electrolyte additives can be used to modify the chemistry occurring at the cathode surface. For example, lithium nitrate additive has been shown to promote the solution-based method of oxygen reduction, thus improving he cycle life of the batteries. Stable pectrolytes also require availability of polar tom to coordinate to Li ins, donor number.

EIS measurements were conducted at directly after the cells assembly, after. the cells were discharged, and after ful recharge. The state of decomposed electrolytes was detected by proton NMR spectroscopy. The samples for NMR analysis were taken at the same three corresponding cells conditions as for the EIS measurements (initial, discharged, and recharged).

Electrolyte candidates were chosen due to their lithium and oxygen solubility, high polarity and their
Li-Oxygen Battery
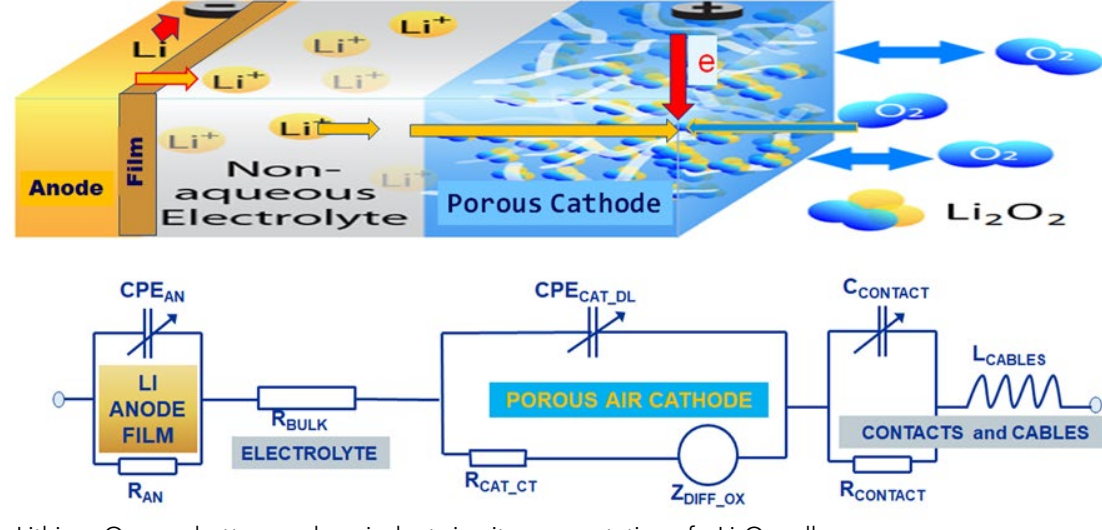

Higher energy density batteries would make a significant difference to our future transportation needs, allowing the development of greener technologies.

desirable features for the above

mentioned lithium-oxygen chemistry.

oxygen would possess high vapour

pressure to prevent rapid evaporation.

The resulting electrolyte candidates were selected from variations of the simple $\mathrm{N}, \mathrm{N}$-dimethylacetamide (DMA) and

urea Tetramethlyurea (MU). Electrolyte solutions were prepared with a mixture of $0.5 \mathrm{M}$ lithium bis(trifluoromethane) sulfonyl imide (LTFSI) and $0.5 \mathrm{M}$ lithiu nitrate $\left(\mathrm{LiNO}_{3}\right)$

A POWERFUL TOOL TO AID THE DEVELOPMENT OF ADVANCED

The NASA researchers concluded that much greater extent than acetamides. $\mathrm{N}, \mathrm{N}$-dimethylpivalamide (TMDMA), a sterically hindered derivative of TMA was the most stable followed by DMA. NMR spectra of DMA and its derivatives showed uncontaminated peaks, in good agreement with literature databases. The urea-derived candidates possessed degradation peaks coresponding to methanol, acetic acid and a methyl-hydroxylamine, while the acetamides showed predominantly methanol. The results demonstrated NMR da for degre between EIS and NMR data for degree of electrolytes' cycling performance.
The development of functional and stable novel electrolytes is a one of battery research. For the lithium-oxygen chemistry, the combination of EIS and NMR provide a powerful tool for analysing the decomposition reactions that occur during repeated discharging and recharging cycles, enabling 作 ectrolytes. The highly aggressive chemical environment of lithium-oxygen batteries results in successively higher charging potentials with every cycle, degradation, and ultimately shorter battery cycle life.

The switch to electric powered ransportation has the potential to fuels, thereby reducing $\mathrm{CO}_{2}$ emissions and their negative effects on climate change. The EIS/NMR analytical approach developed by the NASA researchers will be used to develop dvanced battery components such as optimally designed electrolytes and cathodes with well-structured igherture to provide substantially higher specific energy and longer battery cycle life. Higher energy density batteries will make a significant difference to our future transportation needs, allowing the development of greener high performance electric vehicles. 


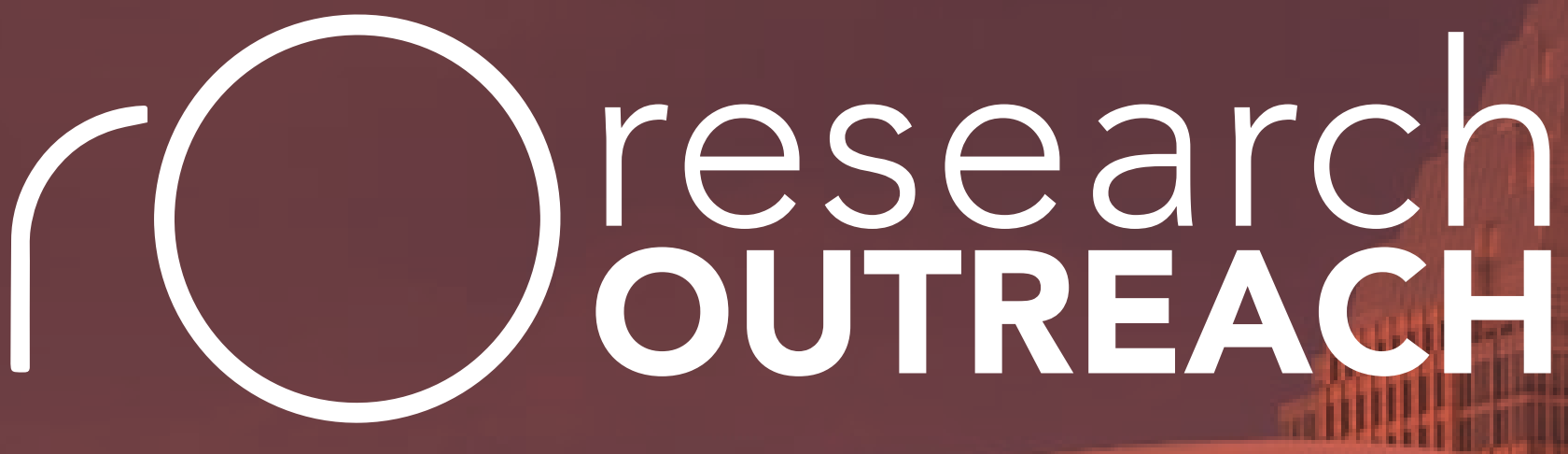

The public outreach magazine for the research community

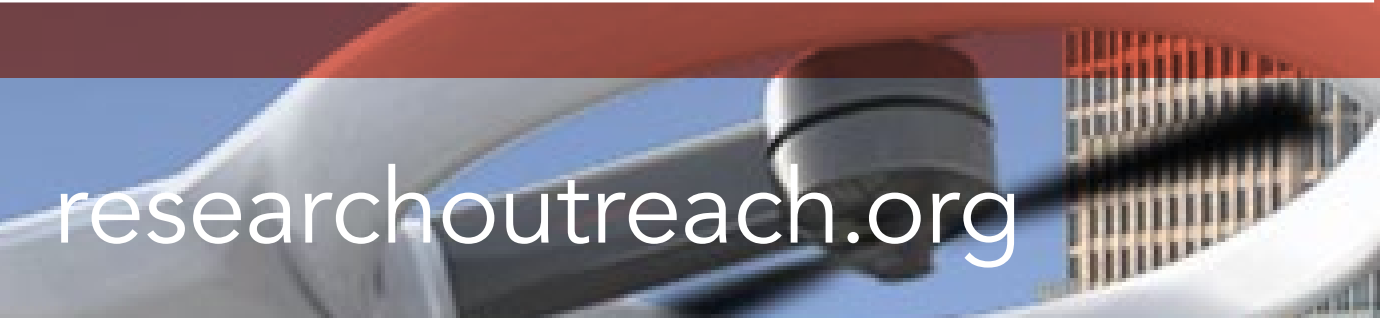

Partnership enquiries: simon@researchoutreach.org

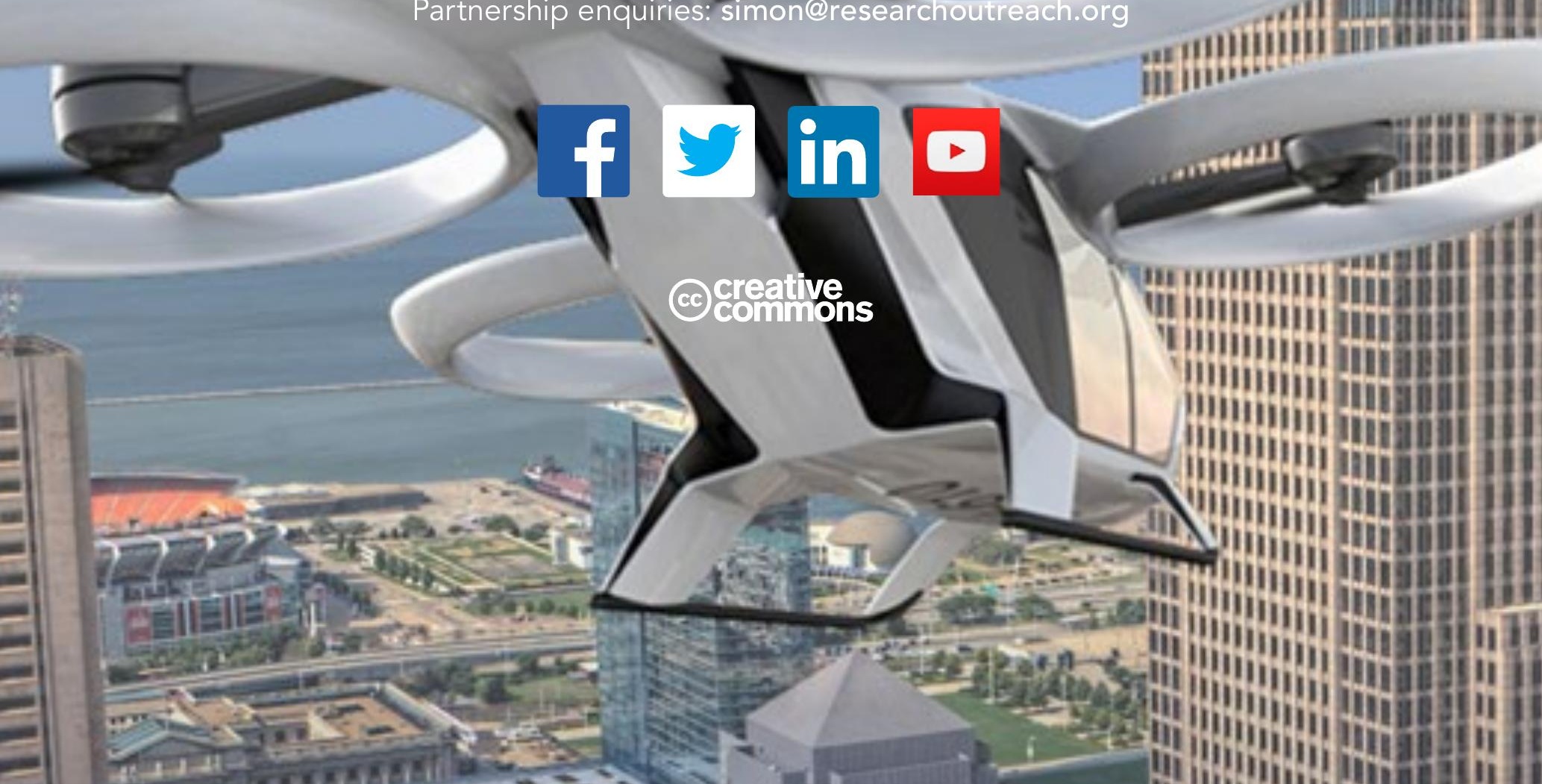

